\title{
SOLUTION OF HOMOGENEOUS LINEAR DIFFERENCE EQUATIONS
}

\author{
J. D. LOVE
}

(Received 31 May 1978)

(Revised 8 March 1979)

\begin{abstract}
When the first two elements of a sequence satisfying a second order difference equation are prescribed, the remaining elements are evaluated from a continued fraction and a simple product.
\end{abstract}

\section{Introduction}

The sequence $u_{n}$ satisfies the second order, linear difference equation

$$
H(n) u_{n+1}+G(n) u_{n}+F(n) u_{n-1}=0, \quad n=1,2, \ldots,
$$

where the $F(n), G(n)$ and $H(n)$ are arbitrary functions of $n$. We seek the solution for all $n$ when $u_{0}$ and $u_{1}$ are prescribed.

If one of the complementary solutions of (1.1) can be found by inspection, or if it is prescribed, Brand [1] shows how the second complementary solution is obtained. Let $v_{n}$ be the known solution, then the second solution $w_{n}$ is generated by setting $u_{n}=v_{n} w_{n}$ in (1.1). The procedure is analogous to finding the second solution of a differential equation when one solution is known. A linear combination of $v_{n}$ and $w_{n}$ is then used to satisfy the prescribed values of $u_{0}$ and $u_{1}$.

The solution given by Brand was established earlier by Funk [3], but the reference is not so readily available. Funk also solves (1.1) when $u_{0}=0$ and $u_{N+1}=0$, so that only $N$ equations are involved. His solution for $u_{1}$ is expressed as the ratio of two tridiagonal determinants, according to Cramer's rule, and he goes on to show that each tridiagonal determinant is expressible as a continued fraction containing the $F(n), G(n)$ and $H(n)$. 
An even earlier solution of (1.1) is given by Perron [4] in terms of a tridiagonal determinant for the case $u_{0}=0, u_{1}=1$ and $H(n)=-1$ for all $n$. He gives

$$
u_{n+1}=C_{1}^{n}, \quad n=1,2, \ldots,
$$

where $C_{1}^{n}$ is defined by (1.4). Perron then points out, like Funk, that $C_{1}^{n}$ is equivalent to a continued fraction.

More recently, Brown [2] has given the generai soiution to (1.i) as a linear combination of $u_{0}$ and $u_{1}$ according to

$$
u_{n+1}=(-)^{n}\left\{u_{0} F(1) C_{2}^{n}+u_{1} C_{1}^{n}\right\} / \prod_{r=1}^{n} H(r) \quad n=2,3, \ldots,
$$

where $C_{1}^{n}$ and $C_{2}^{n}$ are tridiagonal determinants of orders $n$ and $n-1$ defined by

$$
C_{m}^{n}=\left|\begin{array}{ccccccc}
G(m) & H(m) & 0 & \ldots & 0 & . & . \\
F(m+1) & G(m+1) & H(m+1) & \ldots & 0 & . & . \\
0 & F(m+2) & G(m+2) & \ldots & H(n-3) & 0 & . \\
. & 0 & F(m+3) & \ldots & G(n-2) & H(n-2) & 0 \\
. & . & . & \ldots & F(n-1) & G(n-1) & H(n-1) \\
. & . & . & \ldots & 0 & F(n) & G(n)
\end{array}\right|
$$

This result is the generalization of Perron's solution, and clearly reduces to (1.2) when his conditions are satisfied.

Our motivation is to propose an alternative solution to (1.1), which is as general as Brown's results, but which is simpler and avoids the evaluation of two determinants.

\section{Method of solution}

The first step in our method of solution is to reduce (1.1) to a first-order equation by the following rearrangement

$$
\left[\frac{u_{n+1}}{u_{n}}\right]+\frac{G(n)}{H(n)}+\frac{\dot{F(n)}}{\left[\frac{u_{n}}{u_{n-1}}\right]}=0
$$

This is now a nonlinear, first order difference equation for the ratio $u_{n+1} / u_{n}$, which is solved by repeated iteration. The solution is expressed as

$$
\frac{u_{n+1}}{u_{n}}=p_{n},
$$


where $p_{n}$ is defined by the continued fraction

$$
\begin{aligned}
p_{n}=-\frac{G(n)}{H(n)}-\frac{F(n)}{\frac{H(n)}{G(n-1)}-\frac{F(n-1)}{H(n-1)}} & \\
\frac{-\frac{G(n-2)}{H(n-2)}-\ldots}{\cdots} & \frac{-\frac{F(1)}{H(1)}}{\frac{u_{1}}{u_{0}}} .
\end{aligned}
$$

When $n=0$, we have $p_{0}=u_{1} / u_{0}$. Thus each $p_{n}$ as evaluated from (2.3) determines the ratio of successive $u_{n}$, that is $u_{n+1}=p_{n} u_{n}$.

The second step is to use an identity to express $u_{n+1}$ as a product of ratios of successive $u_{n}$

$$
u_{n+1}=\frac{u_{n+1}}{u_{n}} \frac{u_{n}}{u_{n-1}} \ldots \frac{u_{1}}{u_{0}} u_{0}
$$

Substituting from (2.2), the general solution for $u_{n+1}$ is given by

$$
u_{n+1}=u_{0} \prod_{r=0}^{n} p_{r}
$$

The evaluation of the product is simplified if we observe from (2.2) and (2.3) that successive $p_{n}$ satisfy

$$
p_{n}=-\frac{G(n)}{H(n)}-\frac{F(n)}{H(n)} \frac{1}{p_{n-1}}, \quad n=1,2, \ldots
$$

The solution given by (2.5) is formally identical to (1.3), as may be verified, but has the advantage that only one set of calculations is required to evaluate the $p_{n}$, whereas two sets of calculations must be employed to determine $C_{1}^{n}$ and $C_{2}^{n}$ in (1.3).

\section{Solutions when $u_{0}$ or $u_{1}$ vanish}

If both $u_{0}$ and $u_{1}$ are non-zero, $u_{n+1}$ is given by (2.5), and if both $u_{0}$ and $u_{1}$ vanish, there is only the trivial solution $u_{n}=0$ for all $n$. The two cases of interest occur when either $u_{0}$ or $u_{1}$ vanishes. When $u_{0}=0$, the identity (2.4) is contracted 
by removing the dependence on $u_{0}$. Consequently, (2.5) is replaced by

$$
u_{n+1}=u_{1} \prod_{r=1}^{n} p_{r}, \quad n=1,2, \ldots
$$

Similarly, when $u_{1}=0$, the identity (2.4) is contracted by removing the dependence on both $u_{0}$ and $u_{1}$. Hence

$$
u_{n+1}=u_{2} \prod_{r=2}^{n} p_{r}, \quad n=2,3, \ldots,
$$

and $u_{2}$ is expressed in terms of $u_{0}$ via (1.1)

$$
u_{2}=-\frac{F(1)}{H(1)} u_{0}
$$

Putting (3.3) into (3.2)

$$
u_{n+1}=-\frac{F(1)}{H(1)} u_{0} \prod_{r=2}^{n} p_{r}, \quad n=2,3, \ldots
$$

\section{References}

[1] L. Brand, Differential and difference equations (Wiley, New York, 1966), p. 363.

[2] A. Brown, "Solution of a linear difference equation", Bull. Austral. Math. Soc. 11 (1974), 325-331.

[3] P. Funk, Die linearen Differenzengleichungen und ihre Anwendung in der Theorie der Baukonstruktionen (Springer, Berlin, 1920), p. 26.

[4] O. Perron, Die Lehre von den Kettenbrüchen (Teubner, Leipzig, 1913), p. 11.

Department of Applied Mathematics

Australian National University

Canberra A.C.T., 2600

Australia 